Gemeinfreiheit, Kollektiv und Kulturallmende - Uploadfilter schaffen neue „Tipping Points“ in der Kulturindustrie

\title{
Marion Goller
}

Am 06.06.2019, also am zwanzigsten Tag nach ihrer Veröffentlichung im Amtsblatt der Europäischen Union, ist die Richtlinie 2019/790/EU ${ }^{1}$ in Kraft getreten. Den Mitgliedstaaten bleiben ab diesem Zeitpunkt zwei Jahre, um die Richtlinie in nationales Recht umzusetzen. Die vor der Verabschiedung vieldiskutierten Uploadfilter werden also noch einige Zeit Teil der Debatte bleiben. Der folgende Beitrag untersucht verschiedene mögliche Auswirkungen, die Uploadfilter in der Zukunft haben könnten. Er richtet sich dabei nicht ausschließlich an ein rechtswissenschaftliches $\mathrm{Pu}$ blikum. Im ersten Teil wird daher noch einmal zusammengefasst, inwieweit die DSM-Richtlinie die Verwendung von Uploadfiltern vorschreibt, und es wird kurz auf eine Alternative eingegangen, welche statt der Filter denkbar gewesen wäre. Der zweite Teil beleuchtet drei potenzielle Konsequenzen, die nicht unbedingt auf den ersten Blick ins Auge fallen und die auch nicht notwendigerweise zusammenhängen, sondern unabhängig voneinander eintreten können. Diese betreffen den Umgang mit gemeinfreien Werken, das Verhältnis von Urheber:innen zu Verwerterunternehmen und Verwertungsgesellschaften und die Entwicklung von sog. Kulturallmenden, also von der gemeinschaftlichen Verwaltung kreativer Inhalte. Der Beitrag soll aufzeigen, welche unterschiedlichen - teilweise gegensätzlichen - Entwicklungen hier durch die Uploadfilter angestoßen werden können.

1 Richtlinie (EU) 2019/790 des Europäischen Parlaments und des Rates vom 17. April 2019 über das Urheberrecht und die verwandten Schutzrechte im digitalen Binnenmarkt und zur Änderung der Richtlinien 96/9/EG und 2001/29/EG, nachfolgend DSM-Richtlinie. 


\section{Vorüberlegungen zu Uploadfiltern}

Mit der Verabschiedung der DSM-Richtlinie hat der EU-Gesetzgeber eine Grundregel des Internet aufgegeben, ohne welche das partizipative Web 2.0 nicht in seiner heutigen Form hätte entstehen können: Plattformen haften grundsätzlich nicht unmittelbar für Nutzerinhalte. In den USA ist dieser Grundsatz für das Urheberrecht im $\mathrm{DMCA}^{2}$ niedergelegt, für andere Rechtsverletzungen in Section 230 CDA, den Kettemann kürzlich als „das wichtigste Internetgesetz der Welt" bezeichnete. ${ }^{3}$ Die EU ${ }^{4}$ hat den Grundsatz in Art. 14 der Richtlinie 2000/31/EG ${ }^{5}$ kodifiziert, in Deutschland umgesetzt durch $\$ \$$ 8- 10 TMG und konkretisiert durch die Rechtsprechung 6 .

\subsection{Haftung der Plattformen}

In Artikel 17 der DSM-Richtlinie wird nun diese Grundregel ausdrücklich für Urheberrechtsverletzungen außer Kraft gesetzt. Stattdessen werden Plattformen so bewertet, als ob sie selbst die urheberrechtlich relevanten Handlungen der öffentlichen Wiedergabe oder öffentlichen Zugänglichmachung vornehmen, wenn ein:e Nutzer:in geschützte Inhalte teilt. Bedenkt man die schiere Menge an Inhalten, die täglich in sozialen Medien geteilt werden, wird schnell klar, dass eine Überprüfung derselben unmöglich von Menschen geleistet werden kann. ${ }^{7}$ Es müssen also automatische Systeme bereitgehalten werden, welche in der Lage sind, verletzendes Ma-

2 Digital Millennium Copyright Act, dort in 17 U.S. Code $\$ 512$.

3 Hans-Bredow-Institut, Trumps Gesetz gegen Soziale Medien, https://www.hans-bred ow-institut.de/de/blog/trumps-gesetz-gegen-soziale-medien (zuletzt abgerufen am 20.07.2020).

4 Damals noch EG.

5 Auch bekannt als eCommerce-Richtlinie.

$6 \mathrm{Zu}$ den Gemeinsamkeiten und Unterschieden der Intermediärshaftung im Äußerungs-, Urheber-, Marken- und Lauterkeitsrecht siehe Hofmann ZUM 2017, 102, 103.

7 Facebook hat zurzeit 2,7 Milliarden aktive Nutzer:innen, siehe https://www.statista .com/statistics/264810/number-of-monthly-active-facebook-users-worldwide/ (zuletzt abgerufen am 20.07.2020). Auf YouTube wurden im Mai 2019 durchschnittlich in jeder Minute 500 Stunden Videomaterial hochgeladen, siehe https:// www.statista.com/statistics/259477/hours-of-video-uploaded-to-youtube-every-minu te/ (zuletzt abgerufen am 20.07.2020). 
terial zu erkennen. ${ }^{8} \mathrm{Da}$ „Uploadfilter“ im Koalitionsvertrag eigentlich abgelehnt worden waren, verband die deutsche Bundesregierung ihre $\mathrm{Zu}$ stimmung im Rat mit einer Protokollerklärung, dass diese „nach Möglichkeit zu verhindern" seien.

Bis heute ist unklar, wie das möglich sein soll. ${ }^{10}$ Artikel 17 Abs. 4 der DSM-Richtlinie sieht einen Haftungsausschluss dann vor, wenn die Plattform „alle Anstrengungen unternommen hat, um die Erlaubnis einzuholen; und nach Maßgabe hoher branchenüblicher Standards für die berufliche Sorgfalt alle Anstrengungen unternommen hat, um sicherzustellen, dass bestimmte Werke und sonstige Schutzgegenstände, zu denen die Rechteinhaber den Anbietern dieser Dienste einschlägige und notwendige Informationen bereitgestellt haben, nicht verfügbar sind“.

Diese Bereitstellung notwendiger Informationen durch die Rechteinhaber ${ }^{11}$ meint nichts Anderes als die Übergabe von Referenzdateien, die für die Konfiguration automatischer Filtersysteme vonnöten sind. Mehr noch, wer im Zuge der Debatte dem Berichterstatter Axel Voss, der die Reform maßgeblich zu verantworten hatte, eine Protest-E-Mail schrieb, erhielt als Antwort einen Standardtext, der ausdrücklich auf Filtersysteme Bezug nimmt: „Um den Schutz der urheberrechtlichen Werke zu gewährleisten, sollen die Plattformen aufgrund der Informationen, die die Rechteinhaber zur Verfügung stellen müssen, sicherstellen, dass die Plattformen erkennen können, dass es sich um ein geschütztes Werk handelt. Hierfür wird Erkennungssoftware eingesetzt, die seit ca. 10 Jahren bereits existiert und zum Beispiel von YouTube auf freiwilliger Basis eingesetzt wird (ohne dass es bis heute eine Anti-Zensur-Kampagne ausgelöst hat)."12 Tatsächlich läuft die Anti-Zensur-Kampagne der Electronic Frontier Foundation gegen

8 Früher oder später wird sich wahrscheinlich der EuGH mit der Frage befassen müssen, ob die DSM-Richtlinie nicht wegen dieser Filterpflichten gegen die Unionsgrundrechte verstößt, vgl. Spindler CR 2020, 50.

9 Interinstitutionelles Dossier: 2016/0280(COD), 7986/19 ADD 1 REV 2, siehe https://eur-lex.europa.eu/legal-content/DE/TXT/PDF/?uri=CONSIL:ST_7986_2019 _ADD_1_REV_2\&from=EN (zuletzt abgerufen am 20.07.2020).

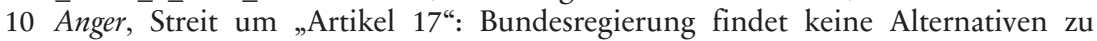
Uploadfiltern, https:/www.handelsblatt.com/politik/deutschland/eu-urheberrech t-streit-um-artikel-17-bundesregierung-findet-keine-alternativen-zu-uploadfiltern/2 5020802.html (zuletzt abgerufen am 24.09.2020).

11 Mit dem Wort „Rechteinhaber“ sind überwiegend Unternehmen und nicht natürliche Personen gemeint. Daher wird es in diesem Text wird - wie in der Richtlinie - in der männlichen Form genutzt, genau wie „Hersteller“ oder „Betreiber“.

12 Auszug aus der Antwort von Axel Voss auf Protest-E-Mails. 
YouTubes Filtersystem bereits seit dessen Einführung. ${ }^{13}$ Der Bezug auf „von Rechteinhabern bereitgestellte Informationen“" in der Richtlinie weist darauf hin, dass hier das Content-ID-System Pate gestanden hat. Was einige Akteure bereits freiwillig eingeführt haben, wird nun für alle verpflichtend. ${ }^{14}$

\subsection{Ausschließlichkeit statt reiner Vergütungspflicht}

Ziel der Reform war und ist es, die Rechteinhaber bei Verhandlungen mit den Plattformen in eine stärkere Position zu bringen. Die Big Three der Musikindustrie $^{15}$ etwa haben bereits Lizenzverträge mit YouTube. Dank der Haftungsprivilegierung konnte der Videodienst diese allerdings dazu bringen, nur geringe Beträge pro Stream zu akzeptieren, weil die Alternative gewesen wäre, völlig leer auszugehen. Dieses Ungleichgewicht führt nach Ansicht der Rechteinhaber zum vielbeklagten „value gap“, also dem Phänomen, dass YouTube mit geschützten Inhalten hohe Werbeeinnahmen generiere, aber die Rechteinhaber nur unzureichend daran beteilige. ${ }^{16}$ Der Wegfall der Haftungsprivilegierung soll also nicht die Inhalte von der Plattform „filtern“, sondern eine höhere Vergütung ermöglichen. ${ }^{17}$

Fraglich ist, ob es hierzu unbedingt der Ausweitung des Ausschließlichkeitsrechts der öffentlichen Wiedergabe oder Zugänglichmachung auf Handlungen Dritter bedurft hätte. Das Urheberrecht kennt verschiedene

13 Lohmann, YouTube's Content ID (C)ensorship Problem Illustrated, https://www. eff.org/de/deeplinks/2010/03/youtubes-content-id-c-ensorship-problem (zuletzt abgerufen am 20.07.2020).

14 Wimmers / Barudi, GRUR 2017, 327, 334; Hofmann bezweifelt daher, dass sich durch die Richtlinie überhaupt viel ändern wird, siehe Hofmann GRUR 2019, 1219, 1222-1223.

15 Die Labels Sony Music Entertainment, Universal Music Group und Warner Music Group teilen fast zwei Drittel des weltweiten Musikmarktes unter sich auf, siehe https:/www.statista.com/statistics/422926/record-companies-market-share-w orldwide-physical-digital-revenues/ (zuletzt abgerufen am 20.07.2020).

16 Dredge, Why is the music industry battling YouTube and what happens next?, https://www.theguardian.com/technology/2016/may/20/music-industry-battling-g oogle-youtube-what-happens-next (zuletzt abgerufen am 20.07.2020); kritisch zur Figur des „value gap“ Nolte ZUM 2017, 304; Wimmers / Barudi GRUR 2017, 327; dazu wiederum skeptisch Metzger ZUM 2018, 233, 237-238.

17 Zur Frage, wie praktikabel diese Regelung für nicht-professionelle Urheber:innen sein wird, siehe Spindler CR 2020, 50, 52 und Hofmann GRUR 2019, 1219, 12251226. 
Schranken, welche die grundsätzlichen Verbote durchbrechen und vorsehen, dass für bestimmte Nutzungen keine gesonderte Erlaubnis einzuholen ist. Auch diese Nutzungen erfolgen keineswegs kostenlos. Die Bibliothekstantiemen und die Vergütung für die Privatkopie werden von den dafür geschaffenen Verwertungsgesellschaften erhoben. ${ }^{18}$ Im Musikgeschäft haben diese sogar noch größere Verantwortung. Hier wurde der GEMA die Aufgabe zugewiesen, die gesamte Zweitverwertung für die Komponist:innen und Texter:innen abzuwickeln, da es schlicht zu aufwändig wäre, wenn ein Radiosender oder eine Cover-Band für jeden gespielten Song die Erlaubnis bei den jeweiligen Musikurheber:innen einzuholen hätte. Sogar die Vergütung der Aufnahme eines Werks auf einen Tonträger läuft über die GEMA. ${ }^{19}$

Viel hätte dafür gesprochen, die Verwendung urheberrechtlich geschützter Inhalte durch die Nutzer:innen von Plattformen als Zweitverwertung einzuordnen und die Vergütung entsprechend zu gestalten. Anstatt für jede Nutzung die Erlaubnis einzuholen, müssten diejenigen Parteien, die mit der Verwendung geschützter Inhalte Einnahmen generieren, einen Teil dieser Einnahmen abgeben. Ähnlich wie die Hersteller von Kopiertechnologie würden Plattformbetreiber zuvor ausgehandelte Beträge an die Verwertungsgesellschaften bezahlen, die diese an ihre Mitglieder ausschütten. ${ }^{20}$ Das scharfe Schwert der zwangsweisen Filter wäre vermieden worden. Die Nutzer:innen hätten bei der Verwendung geschützter Medieninhalte Rechtssicherheit und wüssten außerdem, dass die Urheber:innen dieser Inhalte vergütet werden. Die erfolgreichen unter ihnen würden durch ihre generierten Einnahmen zu dieser Vergütung beitragen. Vor allem aber könnten die Schranken des Urheberrechts gewahrt werden. Laut Artikel 17 Abs. 7 Unterabsatz 2 der DSM-Richtlinie sollen sich die Nutzer:innen auch weiterhin auf diese berufen können. Den Algorithmen wird man die Unterscheidung, ob eine Nutzung als Zitat oder Parodie zulässig ist, so bald nicht beibringen können. ${ }^{21}$

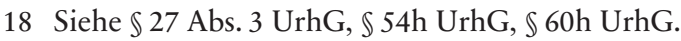

$19 \$ 1$ des GEMA-Berechtigungsvertrags in der Fassung vom 25.05.2019.

20 Ein Vorschlag für ein zweistufiges Modell, das eine Vergütungspflicht mit einer Zwangslizenz verbindet, findet sich bei Leistner / Metzer, Wie sich das Problem illegaler Musiknutzung lösen lässt, https://www.faz.net/aktuell/feuilleton/medien/g ema-youtube-wie-sich-urheberrechts-streit-schlichten-liesse-14601949.html (zuletzt abgerufen am 20.07.2020); ähnlich schon Leistner / Metzger IIC 2017, 381, 383-384.

21 Becker ZUM 2019, 636, 644; Lobo, Streit über die Urheberrechtsreform - Wollt ihr Europa zerstören?, https:/www.spiegel.de/netzwelt/netzpolitik/urheberrechtsrefo rm-wollt-ihr-europa-zerstoeren-a-1252993.html (zuletzt abgerufen am 20.07.2020); 
Zweifellos war der Einsatz solcher Filtersysteme ohnehin nicht mehr aufzuhalten, sondern Teil der allgemeinen Automatisierung, die in immer mehr Lebensbereiche Einzug hält. Schon jetzt verfügen zumindest alle großen Plattformen über Mechanismen, welche ihnen erlauben, die nutzer:innenseitig generierten Inhalte auszuwerten und zu kategorisieren. Basierend auf diesen Informationen geben sie Empfehlungen für weitere Inhalte ab mit dem Ziel, mehr engagement und dadurch mehr Werbeeinnahmen zu erzeugen. Der Staat könnte die Nutzung solcher Filter kritisch beobachten und - wenn nötig - Maßnahmen ergreifen, um ihren Missbrauch zu erschweren und tiefgreifende Einschränkungen der Meinungsund Informationsfreiheit zu verhindern. Stattdessen wird ihre Verwendung nun zwingend vorgeschrieben. Wie schon bei den zuvor in diesem Bereich ergangenen EU-Rechtsakten erfährt das Urheberrecht keine bloße Vereinheitlichung, sondern eine Stärkung. ${ }^{22}$ Das Urheberrecht, welches in Ermangelung einer alternativen Vision ,als zentrales Wirtschaftsrecht des Internets ${ }^{\text {"23 }}$ zweckentfremdet wurde, rangiert an erster Stelle. ${ }^{24}$

Beuth, Umstrittene Urheberrechtsreform - Woher die Uploadfilter kommen könnten, https:/www.spiegel.de/netzwelt/web/upload-filter-fuer-die-urheberrecht sreform-wer-entwickelt-die-software-a-1261320.html (zuletzt abgerufen am 20.07.2020); Kastl GRUR 2016, 671, 675.

22 Die Richtlinie 91/250/EWG (Computerprogramm-Richtlinie), abgelöst durch Richtlinie 2009/24/EG, schrieb vor, dass Computerprogramme dem Urheberrechtsschutz unterfallen müssen. Die Richtlinie 96/9/EG führte ein Schutzrecht für Datenbanken ein. Die Richtlinien 93/98/EWG und 2011/77/EU verlängerten die Schutzfristen für das Urheberrecht bzw. verwandte Schutzrechte. Die InfoSocRichtlinie 2001/29/EG sieht zwingend vor, dass die Nutzungsart des öffentlich Zugänglichmachens den Rechteinhabern vorbehalten ist, überlässt es aber den Mitgliedstaaten, ob und inwieweit sie auch die - abschließend aufgeführten Schranken des Urheberrechts umsetzen wollen. Kritisch zu dem so kreierten Ungleichgewicht Stieper in ZUM 2019, 393, 394.

23 Becker ZUM 2019, 636, 648.

24 Das Urheberrecht ist damit auch sehr viel stärker geschützt als etwa das Persönlichkeitsrecht, welches ebenfalls mit der Meinungs- und Informationsfreiheit konfligieren kann. Nicht einmal das viel kritisierte NetzDG enthält eine Verpflichtung zum automatischen Herausfiltern von beleidigenden und/oder rassistischen Begriffen. Hier obliegt es immer noch den Nutzer:innen selbst, rechtswidrige Inhalte zu melden. Normalerweise ist dann eine Stellungnahme einzuholen. Nur wenn die Rechtswidrigkeit offensichtlich ist, muss gemäß $₫ 3$ Abs. 2 Nr. 2 NetzDG binnen 24 Stunden gelöscht werden. 


\section{Tipping Points}

Diese Entwicklung kann nun verschiedene Konsequenzen haben, die konträr zueinander stehen:

(1) Die Automatisierung kann erstens die Gemeinfreiheit weiter einschränken oder zu neuer Bedeutung erstarken lassen.

(2) Sie kann zweitens den Kreativen mehr Unabhängigkeit geben, aber gleichzeitig die Verwertungsgesellschaften, welche diese vertreten, schwächen oder gar obsolet machen.

(3) Und das neue Bewusstsein um Sinn und Grenzen des Urheberrechts, welches durch die Debatte um die Uploadfilter ausgelöst wurde, kann drittens die Aufmerksamkeit für alternative Möglichkeiten zur Finanzierung und Vermarktung kreativer Arbeit erhöhen - oder aber die Uploadfilter können die Verbote zementieren und ihre Hinterfragung bremsen.

\subsection{Schwächung oder Stärkung der Gemeinfreibeit}

Der erste Tipping Point betrifft den Umgang mit gemeinfreien Werken und Inhalten.

Das Internet wird häufig als Sphäre abseits jeglicher Kontrolle beschrieben. Das Stichwort „rechtsfreier Raum” bezieht sich nicht nur auf den Handel mit verbotenen Waren oder auf die Verbreitungen von Unwahrheiten oder Schmähungen, sondern auch auf Verletzungen des Urheberrechts. ${ }^{25}$ Die Verschärfungen, die das Urheberrecht seit der Jahrtausendwende durch verschiedene Reformen erfahren hat, sollen diesen Kontrollverlust ausgleichen. Zu denken ist etwa an $\$ 19$ a UrhG ${ }^{26}$, der dem Rechteinhaber das ausschließliche Recht der öffentlichen Zugänglichmachung zuweist, an $\$ 101 \mathrm{UrhG}^{27}$, der dem Rechteinhaber einen Auskunftsan-

25 Freund, „Das Internet ist ein rechtsfreier Raum“, Interview mit Alexander Skipis vom Börsenverein des Deutschen Buchhandels, https://www.welt.de/kultur/articl e3558839/Das-Internet-ist-ein-rechtsfreier-Raum.html (zuletzt abgerufen am 20.07.2020); Banse, Das Internet - ein rechtsfreier Raum?, https://www.deutschlan dfunk.de/das-internet-ein-rechtsfreier-raum.724.de.html?dram:article_id=99567 (zuletzt abgerufen am 20.07.2020).

26 Vorschrift eingefügt durch das Gesetz zur Regelung des Urheberrechts in der Informationsgesellschaft vom 10.09.2003.

27 Vorschrift eingeführt durch das Gesetz zur Verbesserung der Durchsetzung von Rechten des geistigen Eigentums vom 7. Juli 2008. 
spruch gegen den Internet Service Provider gibt, oder an die Einschränkung des $₫ 53 \mathrm{UrhG}^{28}$, wonach Privatkopien nur von solchen Vorlagen angefertigt werden dürfen, die nicht offensichtlich rechtswidrig öffentlich zugänglich gemacht wurden.

Die Rechteinhaber haben sich aber nicht allein auf den Gesetzgeber verlassen, sondern parallel immer auch versucht, die Vervielfältigung von Inhalten technisch zu unterbinden. Anfangs waren diese Versuche noch von wenig Erfolg gekrönt. Jeder Kopierschutz wurde bald geknackt und Ideen wie die „Un-CD“, die in der Stereo-Anlage, aber nicht im Laufwerk am PC abspielbar sein sollte, zogen den Zorn der Kund:innen auf sich..$^{29}$ Obwohl Urheberrechtsverletzungen selbst bereits sowohl verboten als auch strafbar sind, wurde die Umgehung von Kopierschutzmaßnahmen in $\$ 95$ a UrhG ${ }^{30}$ ihrerseits noch einmal gesondert sanktioniert. Gegenwärtig ebbt hierzulande der Widerstand gegen "digitales Rechtemanagement" (DRM) immer mehr ab, was nicht zuletzt dadurch begünstigt wird, dass die meisten Konsument:innen ihren Mediengenuss nicht mehr am frei konfigurierbaren Allzweck-PC ausüben, sondern an speziellen Geräten, bei denen man sich an den durch Kopierschutz reduzierten Funktionsumfang gewöhnt hat oder ihn nie anders kannte. ${ }^{31}$

Die Uploadfilter sind gleichsam DRM für Plattformen. Und wie bei DRM geht die Kontrolle, welche die Rechteinhaber auf technischem Weg gewinnen, weit über dasjenige Maß hinaus, welches "offline“ gegolten hatte. ${ }^{32}$ Wer ohne Uploadfilter ein Urheberrecht durchsetzen will, muss erst einmal nachweisen, dass ihm:ihr dieses überhaupt zusteht. Verletzungen müssen einzeln verfolgt werden; häufig führt der Weg dabei über die Gerichte. Das ist für die Rechteinhaber aufwändig und teuer, aber auch ange-

28 Vorschrift geändert durch das Zweite Gesetz zur Regelung des Urheberrechts in der Informationsgesellschaft vom 26. Oktober 2007.

29 Hansen, Un-CD-Bändiger: Abspielprobleme beseitigen mit unCDcopy, https://ww w.heise.de/ct/artikel/Un-CD-Baendiger-289314.html (zuletzt abgerufen am 20.07.2020).

30 Vorschrift eingefügt durch das Gesetz zur Regelung des Urheberrechts in der Informationsgesellschaft vom 10.09.2003.

31 Ausführlich bei Doctorow, Lockdown, https://boingboing.net/2012/01/10/lockdow n.html (zuletzt abgerufen am 25.09.2020).

32 Mehr zur paradoxen Situation von Wissen im digitalen Zeitalter schon bei Hess/ Ostrom, Overview, in: Hess/Ostrom Knowledge as a Commons, 3, 14; Zur Erläuterung, wie über DRM der Erschöpfungsgrundsatz umgangen wird, vgl. Specht ZGE / IPJ 8 2016, 289. 
messen, denn das Urheberrecht ist ein staatlich verliehenes Exklusivrecht $^{33}$, dessen Durchsetzung auch staatlich kontrolliert werden sollte. Als das Urheberrecht geschaffen wurde, richtete es sich vor allem an Unternehmen. Privatleute hatten gar keine Möglichkeit, Urheberrechte zu verletzen, weil die dazu nötige Technologie ihnen nicht zur Verfügung stand. Das Urheberrecht sollte vor allem den sog. „Nachdruck“ oder „Raubdruck" verhindern ${ }^{34}$, kaum eine Privatperson verfügte aber über eine Druckpresse. Mit der Erfindung und Verbreitung moderner Technologien wie Fotokopierer, Videorekorder, Kassettenrekorder oder CD-Brenner wurde es auch für Privatpersonen möglich, urheberrechtlich geschützte Inhalte zu vervielfältigen. Nachdem die Rechtsprechung diese Handlungen zunächst untersagte ${ }^{35}$, entschied sich der Gesetzgeber bei Schaffung des neuen UrhG im Jahr $1965^{36}$ für eine Erlaubnis mit Vergütungspflicht. Statt eines Verbots wurden Abgaben eingeführt, welche die Urheber:innen für Privatkopien entschädigen sollte. Die Überwachung privater Handlungen wurde als nicht wünschenswert angesehen. Vor allem aber erschien sie nicht durchsetzbar. Bei Schaffung der Schranke der Privatkopie dürfte also die Überlegung ausschlaggebend gewesen sein, dass die allermeisten privaten Nutzungen unerfasst und damit unvergütet bleiben würden, wenn man nicht von den Herstellern eine Abgabe einfordere. ${ }^{37}$

Dank Digitalisierung und Internet sind Vervielfältigung und Verbreitung noch einmal sehr viel simpler geworden. Auch deshalb wird das Internet gerne als „rechtsfreier Raum“ bezeichnet, bzw. gefordert, es dürfe kein solcher sein. Es ist nicht zu leugnen, dass sich durch das Internet das Kräfteverhältnis zwischen Rechteinhabern und Konsument:innen vorerst zugunsten letzterer verschoben hat. Uploadfilter versetzen dieses Verhält-

33 In Deutschland wohl Mindermeinung; hier hat sich die monistische Lehre durchgesetzt, nach welcher naturrechtlich begründete Urheberpersönlichkeitsrechte und Verwertungsrechte einheitlich behandelt werden, vgl. Kreutzer, Regelungsalternativen, 41-43 m.w.N. Derselbe spricht sich für eine Trennung von Urheberschutz und Werkschutz aus, vgl. Kreutzer, Regelungsalternativen, ab 397. Weniger umstritten ist die Einordnung als Exklusivrecht für das US-Copyright; dort ist sogar die Bezeichnung als Monopol nicht selten, vgl. Lessig, Future, 58, oder Boldrin/Levine, Against Intellectual Monopoly, 9. Außerdem spricht die US-Verfassung in Art. I Sec 8 ausdrücklich von exclusive rights.

34 Kublen, Erfolgreiches Scheitern, 68-71 und 147-149.

35 Damals noch unter Geltung des Gesetzes betreffend das Urheberrecht an Werken der Literatur und der Tonkunst (LUG), siehe BGH, Urteil vom 18.05.1955 - I ZR 8/54 - „Grundig-Reporter“, GRUR 1955, 492.

36 Inkrafttreten 1966.

37 Grübler, BeckOK Urheberrecht, $\$ 53$ Rn. 1. 
nis jedoch nicht einfach in den vorherigen Zustand zurück, sondern geben den Rechteinhabern mehr direkte Kontrolle, als diese jemals hatten. Sie müssen nicht länger abmahnen und den Rechtsweg bemühen, sondern können die Nutzung ihrer Inhalte direkt steuern. ${ }^{38}$ Die „Aktionslast“ ${ }^{* 39}$ oder „Angriffs- und Begründungslast “ ${ }^{40}$ wird umgekehrt. ${ }^{41}$ Es muss nicht mehr der Rechteinhaber gegen Verstöße vorgehen, sondern der:die Nutzer:in muss sich wehren, wenn Inhalte zu Unrecht blockiert oder monetarisiert werden. Uploadfilter erlauben die Durchsetzung des staatlich verliehenen Exklusivrechts Urheberrecht ohne staatliches Zutun. Das Potential für Missbrauch ist erheblich. Wer die Filter steuert, kann auch fremde oder gemeinfreie Inhalte beanspruchen ${ }^{42}$; für diese Praxis scheint sich aktuell der Begriff „over-claiming“ oder „Overclaiming“ durchzusetzen. ${ }^{43}$

Der bekannteste der bereits vorhandenen Filter, YouTubes „Content ID“, setzt all das recht nutzer:innnenfeindlich um. Wer sich gegen Content ID claims wehrt und dabei unterliegt ${ }^{44}$, kann nach drei Verstößen gemäß der Nutzungsbedingungen den ganzen Account verlieren. Umgekehrt hat Overclaiming keine solchen Konsequenzen. Die Plattformen haben kein Interesse daran, sich mit großen Rechteinhabern anzulegen. Die Gefahr ist daher groß, dass Overclaiming ohne Konsequenzen bleibt, während Verstöße insbesondere durch einzelne Nutzer:innen, die sich noch keine große Reichweite aufbauen konnten, mit dem Verlust des Accounts geahndet werden.

38 Zur Funktionsweise dieser Filter siehe Goller, Copyfraud und Overclaiming - was beim Umsetzen der EU-Urheberrechtsreform zu beachten ist, https://irights.info/? $\mathrm{p}=29603$ (zuletzt abgerufen am 20.07.2020).

39 Specht GRUR 2019, 253, 259.

40 Becker ZUM 2019, 636, 640.

41 Das ignoriert Hofmann in seiner fünften These, vgl. Hofmann GRUR 2019, 1219, 1223.

42 Auch dies war schon bei DRM möglich; kritisch hierzu Specht, die fordert, dass technische Schutzmaßnahmen nur dann vor Umgehung zu schützen seien, wenn die zu verhindernde Handlung eine Urheberrechtsverletzung wäre, siehe Specht GRUR 2019, 253.

43 Diesen Begriff benutzt auch Google selbst, siehe Google, Clean up incorrect claims, https://support.google.com/youtube/answer/4352063?hl=en (zuletzt abgerufen am 20.07.2020).

44 Der Erfolg eines Vorgehens gegen claims hängt maßgeblich vom Verhalten des angeblichen Rechteinhabers ab. Dieser hat den Prozess weitgehend unter Kontrolle, siehe https://support.google.com/youtube/answer/2797454?hl=de (zuletzt abgerufen am 20.07.2020). 
Aktuell ist die urheberrechtliche Beanspruchung gemeinfreier Inhalte nicht verboten. ${ }^{45}$ Vor Einführung der Uploadfilter war das auch nicht nötig, da die Durchsetzung von Urheberrechten grundsätzlich mit der Überprüfung ihrer Gültigkeit einherging. Unter einem Regime der Filter führt das Fehlen eines Overclaiming-Verbots aber dazu, dass Rechteinhaber nahezu sanktionslos die Nutzung gemeinfreier Kultur durch andere verhindern können. Wer einen gemeinfreien Inhalt für sich reklamiert hat, kann diesen so lange kontrollieren, bis sich jemand beschwert - in einer Umgebung, in der Beschwerden ein nicht unerhebliches Risiko nach sich ziehen. Zwar sieht Art. 17 Abs. 9 der Richtlinie vor, dass der Rechtsschutz garantiert sein muss, wenn Nutzer:innen sich auf Ausnahmen vom Urheberrecht stützen. Darin liegt aber lediglich ein Rechtsbehelf für den Einzelfall, kein allgemeines Verbot der Beanspruchung gemeinfreier Inhalte. Mehr noch, ohne ausdrückliche Regelung ist sogar fraglich, aufgrund welcher Rechtsgrundlage man einen Rechteinhaber auffordern könnte, seinen unberechtigten claim vollständig und nicht nur im konkreten Fall zurückzuziehen, also nicht nur die konkrete Nutzung zu erlauben, sondern die hinterlegte Referenzdatei zu entfernen. An dieser Stelle führen Uploadfilter die Kreativindustrie an einen Tipping Point, was die Gemeinfreiheit angeht: Ohne ein Verbot von Overclaiming bleiben Uploadfilter eine immense Bedrohung für die Nutzung gemeinfreier Inhalte. ${ }^{46}$ Wünschenswert wäre daher eine Regelung, die Overclaiming ausdrücklich verbietet und dieses Verbot auch mit Sanktionen verknüpft. ${ }^{47}$

Voraussetzung dafür wäre aber ein Umdenken, ein neues Bewusstsein für Bedeutung und Wert der Gemeinfreiheit für die Gesellschaft. Weder

45 Allein aus dem Wettbewerbsrecht lässt sich die Schutzrechtsberühmung als irreführende Handlung gemäß $₫ 3$ UWG untersagen.

46 Auf der Plattform Scribd wurde zeitweise der Bericht des Sonderermittlers Robert Mueller gesperrt, siehe Hurtz, Upload-Filter löschen Dutzende Kopien des Mueller-Berichts, https://www.sueddeutsche.de/digital/upload-filter-mueller-berichtscribd-1.4416802 (zuletzt abgerufen am 20.07.2020); Auf Youtube wurde gemeinfreies Material der NASA blockiert, siehe Lee, How YouTube lets content companies "claim" NASA Mars videos, https://arstechnica.com/tech-policy/2012/08/howyoutube-lets-content-companies-claim-nasa-mars-videos/ (zuletzt abgerufen am 20.07.2020). Der Content ID Filter markiert auf Youtube außerdem regelmäßig gemeinfreie Musikaufnahmen, sodass Videos, die diese verwenden, nicht unter eine CC-Lizenz gestellt werden können, siehe Kaiser, Von einem, der auszog, das Fürchten zu lernen..., https://blog.wikimedia.de/2018/08/06/von-einem-der-auszo g-das-fuerchten-zu-lernen/ (zuletzt abgerufen am 20.07.2020).

47 Hofmann fordert kein ausdrückliches Verbot des Overclaimings, aber immerhin ausdrückliche Nutzerrechte sowie die Anwendung der Grundsätze über die unberechtigte Schutzrechtsverwarnung, vgl. Hofmann GRUR 2019, 1219, 1227-1228.

https://doi.org/10.5771/9783748910664-35, am 26.04.2023, 12:37:49 
Eigentum noch Immaterialgüterrechte werden schrankenlos gewährt; auch das Urheberrecht hat sowohl Voraussetzungen als auch Grenzen. An bestimmten Ideen und an ausgesuchten Nutzungen - wie dem Zitat - besteht ein Freihaltungsinteresse, welches den Austausch über bestehende und die Schöpfung neuer Werke ermöglichen soll. Auch gilt das Urheberrecht nicht ewig. Die Schutzdauer von „Leben +70 Jahre“, die Europa mittlerweile in zahlreiche andere Rechtsordnungen exportiert hat, wird bisweilen - m.E. zurecht - als exzessiv kritisiert. ${ }^{48}$ Dass überhaupt eine zeitliche Beschränkung existiert, ist aber Ausdruck eines wichtigen Grundsatzes: Die Kreativität der Künstler:innen soll letztlich in das kulturelle Erbe einfließen, das uns allen gehört. Dieser Schatz speist die Kreation neuer Ideen, Interpretationen und Werke. Indem sie den urheberrechtlichen Schutz von Reproduktionen gemeinfreier Werke ausdrücklich untersagte, zeigte die EU bei der Verabschiedung der DSM-Richtlinie ein Bewusstsein für die Bedeutung der Gemeinfreiheit. ${ }^{49}$ Hier wurde - anders als bei den Vorgängerrichtlinien ${ }^{50}$ - nicht auf dem höchsten Schutzlevel harmonisiert, sondern ein Ausgleich der Interessen gesucht.

Nicht alle Stakeholder akzeptieren die Bedeutung der Gemeinfreiheit. Während der Verhandlungen zum GATT ${ }^{51}$ wurde der damalige Vorsitzende der MPAA ${ }^{52}$, Jack Valenti, mit der Aussage zitiert, die zeitliche Begrenzung des Urheberrechts sei "legale Piraterie“ und sollte abgeschafft werden. ${ }^{53}$ Dank DRM und Uploadfiltern wäre dies nun technisch möglich. Ein Verbot von Overclaiming erfordert also eine bewusste Entscheidung für das Freihaltungsinteresse, für die Gemeinfreiheit, für die Ausgestaltung der Rechte Einzelner in einer Weise, die der Allgemeinheit nicht schadet. Eine solche bewusste Entscheidung könnte die Gemeinfreiheit zu neuer Stärke führen. ${ }^{54}$

48 Koreng, Die Schutzfrist als Sichtschutz: Wie das Urheberrecht freies Wissen behindert, https://irights.info/?p=28237 (zuletzt abgerufen am 20.07.2020).

49 Siehe Erwägungsgrund 53 und Artikel 14 der DSM-Richtlinie.

50 Siehe Fußnote 22.

51 Allgemeines Zoll- und Handelsabkommen (englisch General Agreement on Tariffs and Trade, GATT).

52 Motion Picture Association of America.

53 Travis, 15 Berkeley Tech. L.J. 777 (2000) at 829.

54 Denkbar wäre ein Verbandsklagerecht wie im UWG, ähnlich auch Spindler CR 2020, 50, 58 . 


\subsection{Kreative zwischen Gatekeepern und Kollektiv}

Der zweite Tipping Point betrifft die Abhängigkeit Kreativschaffender von Verwertern und Verwertungsgesellschaften. Schon lange sind Herstellung und Vertrieb von digitalisierbaren Werken kostengünstiger und weniger aufwändig geworden als sie es im 20. Jahrhundert noch waren. Autor:innen können Bücher per self-publishing ans Publikum bringen; Musiker:innen können ihre Stücke selbst in immer besserer Qualität aufnehmen und vertreiben. Dass trotzdem nicht alle Autor:innen und Musiker:innen die Zusammenarbeit mit Verlagen und Labels beenden, dürfte daran liegen, dass die Vermarktung der eigenen Werke immer noch sehr aufwändig ist, zumal die Konkurrenz eher zu- als abnimmt. Auch die Durchsetzung der Rechte gegenüber etwaigen Verletzer:innen fällt einem Label oder Verlag leichter als dem:der Künstler:in selbst. Allerdings setzt diese Zusammenarbeit meist eine Übertragung dieser Rechte voraus. Verlage und Labels wollen oft nicht in die Vermarktung von Inhalten investieren, die ihnen nicht auch gehören - und das möglichst für die gesamte Schutzdauer der Urheberrechte.

Mit Einführung der Uploadfilter stellt sich nun die Frage, wer diese letztlich steuern wird. Oder, um es mit den Worten der Richtlinie zu sagen: Wer wird „den Anbietern dieser Dienste einschlägige und notwendige Informationen" geben, anhand derer diese die geschützten Werke herausfiltern können? Auf YouTube ist die Teilnahme an Content ID aktuell nur solchen Unternehmen möglich, die eine große Zahl von Urheberrechten innehaben. ${ }^{55}$ Aus der Richtlinie geht auch nicht zwingend hervor, dass die Filter allen zur Verfügung stehen müssen.

Im Bereich Musik steht unabhängigen Künstler:innen die Zusammenarbeit mit speziellen Dienstleistungsunternehmen offen, welche sich auf die Auswertung im Internet spezialisiert haben. Diese Dienstleister behalten einen Teil der Einnahmen, verlangen aber keine Rechteübertragung. Denkt man diese Entwicklung weiter, so könnte die Automatisierung irgendwann dazu führen, dass Inhalte in riesigen Datenbanken als Referenzdateien abgelegt sind, die als weltweite "clearing houses“ oder „one stop shops" fungieren. Potentielle Verwerter können dort schnell und flexibel Lizenzverträge direkt mit den Urheber:innen abschließen. Diese müssten

55 „Dein Kanal muss mindestens 1.000 Abonnenten und bei öffentlichen Videos eine gültige Wiedergabezeit von mehr als 4.000 Stunden erreicht haben." Google, YouTube-Partnerprogramm: Überblick und Voraussetzungen, https://support.goo gle.com/youtube/answer/72851(zuletzt abgerufen am 20.07.2020). 
ihre Rechte nicht länger an Verlage oder Labels übertragen. Stattdessen gäbe es Agenturen, die sich ausschließlich um die Vermarktung kümmern und versuchen würden, die Inhalte in anderen Kunstwerken (Filmen, Serien) oder in Werbeanzeigen unterzubringen. In Verbindung mit einer mächtigen Suchmaschine, die unlizenzierten Content sofort erkennt und filtert, wäre den Urheber:innen die absolute Kontrolle über die OnlineNutzung ihrer Werke möglich. So ausgestaltet könnte die Automatisierung letztlich zu mehr Unabhängigkeit für Kreative führen. ${ }^{56}$

Wenig Beachtung hat bislang die Erwägung gefunden, dass Kreative sich nicht nur von den Gatekeepern lösen können, sondern auch von den der Verwertung teilweise vorgeschalteten Verwertungsgesellschaften. Diese haben sich - ebenso wie Verlage, Labels und Filmhersteller - für die Reform eingesetzt. ${ }^{57}$ Sie versprechen sich von den nun folgenden Lizenzverträgen erhebliche Mehreinnahmen, die sie an ihre Mitglieder ausschütten können. Womöglich schaden sie sich damit aber letztlich selbst. Denn wie oben bereits anklang, wurden die Verwertungsgesellschaften geschaffen, um die Vergütung für solche Nutzungen einzunehmen, bei denen Einzelverträge nicht praktikabel wären. Sobald aber alle Nutzungen genau dokumentiert und kontrolliert werden können, wird die Daseinsberechtigung der Verwertungsgesellschaften eher geschwächt als gestärkt. Bibliothekstantieme und Kopierabgabe können aktuell von Gesetzes wegen nur durch eine Verwertungsgesellschaft geltend gemacht werden. Wer an diesen Geldern beteiligt werden will, muss beitreten. Wenn aber in Zukunft der „one stop shop" Wirklichkeit wird, was sollte eine:n Urheber:in daran hindern, die Verwendung unlizenzierter Kopien per Filter zu verhindern und Einzelverträge abzuschließen?

Wer GEMA und VG Wort ohnehin als überflüssig ansieht, dem:der wird diese Aussicht gefallen. Sicher gibt es am System der Verwertungsgesellschaften viel zu kritisieren. Besonders im Streit um die Verlegerbeteiligung müssen sie sich die Frage gefallen lassen, ob sie sich in diesem Interessenkonflikt nicht besser auf die Seite der Urheber:innen hätten stellen sollen als auf die der Verlage. ${ }^{58}$ Die Verwertungsgesellschaften bieten jedoch auch Schutz für kleine Künstler:innen mit geringer Verhandlungsmacht. Unter Geltung der GEMA-Tarife kostet Taylor Swift dasselbe wie

56 Die Nachteile für die Nutzer blieben voraussichtlich dieselben.

57 Gemeinsame Stellungnahme von GEMA, VG Bildkunst, VG Musikedition und VG Wort, https://www.vgwort.de/fileadmin/pdf/allgemeine_pdf/Aktuelle_Informatio n_Lizenzen_vs._Uploadfilter.pdf (zuletzt abgerufen am 20.07.2020).

58 Hintergründe bei Pachali, Kopiervergütung: VG Wort darf nicht pauschal an Verlage ausschütten, https://irights.info/?p=27286 (zuletzt abgerufen am 20.07.2020). 
Rüdiger Bierhorst. ${ }^{59}$ Dank Verteilungsplan erhält sie gewiss ein größeres Stück vom Kuchen. Aber die Kollektiv-Vertretung macht den Umgang mit einem großen Katalog für die Verwerter weniger bürokratisch und gibt unbekannten Künstler:innen mehr Chancen, im Radio gespielt zu werden. Ganz ohne kollektive Rechtewahrnehmung bestünde die Gefahr, dass die erfolgreichsten Urheber:innen die Preise diktieren und der Rest kostenlos lizenzieren muss, um überhaupt wahrgenommen zu werden. ${ }^{60}$

\subsection{Allmende oder Markt}

Der dritte Tipping Point betrifft die „Systemfrage“: Gibt es jenseits staatlicher Aufträge und marktwirtschaftlicher Konkurrenz noch andere Wege, Kultur zu schaffen und zu vertreiben? Auf der Suche nach Alternativen begannen verschiedene Forscher:innen, allen voran Nobelpreisträgerin Elinor Ostrom, die Gemeingüter oder „Allmende“ wiederzuentdecken. Ostroms Werk „Understanding Knowledge as a Commons“61 will die Leser:innen in verschiedenen Essays lehren, Wissen nicht als Ware oder „Geistiges Eigentum“, sondern als Gemeingut zu betrachten, von dessen Pflege alle profitieren sollten.

Das Urheberrecht ist auf diese Idee nicht ausgerichtet, bedarf also der Modifikation durch alternative Lizenzen. Die Freie-Software-Bewegung legte hierfür im Jahr 1989 mit der ersten Version der GNU General Public License $^{62}$ den Grundstein, auf dem Lawrence Lessig und seine Mitstreiter:innen vom Projekt "Creative Commons" aufbauten. ${ }^{63}$ Creative Commons meint nicht nur die Lizenz, sondern insgesamt die kreative Allmende oder Kulturallmende. Wichtigste Verwenderin und wohl erfolgreichste

59 Wer sich jetzt fragt, wer Rüdiger Bierhorst ist: Das ist der Punkt. Für Interessierte: https://youtu.be/b8AMi0_M4dg (zuletzt abgerufen am 20.07.2020).

60 So verhält es sich schon jetzt bei der Verwendung von Musikaufnahmen, da die Labels der Gesellschaft zur Verwertung von Leistungsschutzrechten sehr viel weniger Rechte übertragen als die Urheber:innen der GEMA.

61 Ostrom / Hess, Overview of the Knowledge Commons, in: Ostrom / Hess, Knowledge as a Commons, 3, abrufbar unter http://www.wtf.tw/ref/hess_ostrom_2007.p df (zuletzt abgerufen am 20.07.2020).

62 GNU General Public License v. 1.0, abrufbar unter https://www.gnu.org/licenses/ old-licenses/gpl-1.0 (zuletzt abgerufen am 20.07.2020).

63 Autorenseite von Lawrence Lessig unter https://creativecommons.org/author/lessi g/ (zuletzt abgerufen am 20.07.2020). 
Wissensallmende aller Zeiten ist die Wikipedia ${ }^{64}$; aber auch verschiedene Künstler:innen, wie etwa die Musikerin Amanda Palmer ${ }^{65}$ oder der Autor und Aktivist Cory Doctorow ${ }^{66}$, veröffentlichen ihre Werke als „some rights reserved".

Mit der alternativen Lizenzierung geht häufig die alternative Finanzierung Hand in Hand. Auf der Plattform Patreon ${ }^{67}$ können Nutzer:innen z.B. Künstler:innen oder auch Journalist:innen mit regelmäßigen Zuwendungen fördern. Die Beträge sind nicht unbedingt hoch, dafür kommen aber bis zu $95 \%$ bei den Geförderten an. ${ }^{68}$ Nachdem das Urheberrecht den Kreativschaffenden Unabhängigkeit vom reichen Mäzen gab, verschafft dieses „Miniatur-Mäzenatentum“ ihnen nun Unabhängigkeit vom Markt. Auf Patreon lässt sich außerdem im internen Forum bestaunen, wie offen manche Künstler:innen mit ihren Fans kommunizieren, wie persönlich die Atmosphäre sein kann. Die Basis für diese Allmende sind Vertrauen und Kommunikation.

Bei ihrem Streit mit Spotify kritisierte Taylor Swift die Einstellung, "that music has no value and should be free" ${ }^{69}$ Innerhalb der Markt-Logik hat sie damit absolut Recht und man muss es ihr hoch anrechnen, dass sie sich dabei nicht in erster Linie für sich selbst, sondern für weniger bekannte Kolleg:innen eingesetzt hat. Auf der anderen Seite ist auch das Gegenteil richtig. Musik und Kunst sind so wertvoll, dass sie frei - im Sinne von möglichst frei zugänglich - sein sollten. Für diese Perspektive muss man gedanklich das Marktsystem verlassen, was vielen Kulturschaffenden, die von diesem System ernährt werden, verständlicherweise nicht immer leicht fällt. Die Debatte um die Urheberrechtsreform hat hierzu einen Anstoß gegeben. Sie hat viele Menschen erstmals dazu gezwungen, sich über-

64 https://de.wikipedia.org/wiki/Wikipedia:Lizenzbestimmungen_Creative_Commo ns_Attribution-ShareAlike_3.0_Unported (zuletzt abgerufen am 20.07.2020).

65 Siehe https://shop.amandapalmer.net/pages/welcome (zuletzt abgerufen am 20.07.2020).

66 Doctorow, Why do you give away your books?, https://craphound.com/littlebrothe r/about/\#freedownload/ (zuletzt abgerufen am 25.09.2020).

67 https://www.patreon.com (zuletzt abgerufen am 25.09.2020).

68 Schieferdecker, Einfach mal Mäzen werden, https://www.zeit.de/kultur/musik/2020 $-05 /$ patreon-musiker-crowdfunding-corona/komplettansicht (zuletzt abgerufen am 20.07.2020).

69 Willman, Exclusive: Taylor Swift on Being Pop's Instantly Platinum Wonder... And Why She's Paddling Against the Streams, https:/www.yahoo.com/entertain ment/bp/exclusive--taylor-swift-on-being-pop-s-instantly-platinum-wonder---andwhy-she-s-paddling-against-the-streams-085041907.html (zuletzt abgerufen am 20.07.2020). 
haupt kritisch mit der Materie auseinanderzusetzen und dürfte einige zu der Überzeugung gebracht haben, dass im Namen des Urheberrechts nicht alles erlaubt sein - oder vielmehr nicht alles verboten werden - sollte.

Dennoch haben die Kritiker:innen „verloren“. Die Uploadfilter werden zur Pflicht, die Befürworter eines möglichst starken Urheberrechts haben sich durchgesetzt. Die automatisierte Rechtsdurchsetzung ist außerdem das genaue Gegenteil von Vertrauen und Kommunikation, der Basis der Kulturallmende. Die Uploadfilter machen das unmöglich, was zuvor lediglich verboten war. Sie beschneiden die Handlungsfreiheit der Nutzer:innen sehr viel tiefgreifender als das reine Urheberrecht, das ihnen als Grundlage und Rechtfertigung dient. So gehen Aushandlungsprozesse verloren, die für die Entwicklung und die Entwicklungsfähigkeit des Rechts von immenser Wichtigkeit sind. ${ }^{70}$ Das auf Verbot und Exklusivität ausgerichtete Urheberrecht ist ohnehin denkbar ungeeignet als zentrales Recht des Internet und muss seit Jahren „Aufgaben erfüllen, für die es nicht geschaffen wurde" ${ }^{\text {"71 }}$. Nun wird es auch noch abseits menschlicher Aushandlungsprozesse automatisch durchgesetzt - und dies immer zugunsten der wirtschaftlich ohnehin stärkeren Partei. „Es droht die Gefahr der Anpassung menschlichen Verhaltens an technische Vorgaben - statt umgekehrt. "72

Fraglich ist jedoch, wie viele Menschen diese Rechtsdurchsetzung als Freiheitsverlust empfinden werden. Das Urheberrecht mag das zentrale Wirtschaftsrecht des Internets sein, die durchschnittlichen Nutzer:innen haben jedoch im besten Fall rudimentäre Kenntnisse in diesem Bereich. So sind Konflikte vorprogrammiert. Und wenn eine Kleinunternehmerin sich einer Zahlungsforderung in Höhe von ca. $1.300 €$ ausgesetzt sieht, weil sie ungenehmigt ein Foto auf der eigenen Webseite genutzt hat, wäre es ihr vielleicht lieber, diese Nutzung wäre technisch unterbunden worden. Wer daran gehindert ist, das Recht zu brechen, erleidet auch nicht die Konsequenzen eines Rechtsbruchs. Es ist unklar, wie viele Personen die „Freiheit, rechtswidrig handeln zu können" vermissen werden. ${ }^{73}$ 
Fazit:

(1) Die Uploadfilter stellen eine Bedrohung für die Gemeinfreiheit dar, der es entgegenzuwirken gilt. Eine bewusste Entscheidung in dieser Hinsicht könnte der public domain zu neuer Bedeutung verhelfen.

(2) Die Automatisierung sollte so ausgestaltet werden, dass sie nicht nur den Gatekeepern die Kontrolle gibt, sondern auch den Urheber:innen und Künstler:innen. Diese sollten aber umgekehrt gut überlegen, ob sie die kollektive Rechtewahrnehmung durch Verwertungsgesellschaften aufgeben wollen.

(3) Die Uploadfilter lösen Konflikte durch Technologie statt Kommunikation. Dadurch droht die Debatte zu erlöschen, welche für die Suche nach Alternativen „jenseits von Markt und Staat"74 so wichtig ist. Die Filter verringern also die Wahrscheinlichkeit, dass in naher Zukunft mehr Menschen Kulturgüter als Gemeingut, in Form einer Allmende, finanzieren und verwalten werden, anstatt sie wie gewohnt als Ware zu konsumieren.

\section{Literatur}

Ablberg, Hartwig / Götting, Horst-Peter: BeckOK Urheberrecht, 27. Aufl., München 2020.

Anger, Heike, Streit um „Artikel 17“: Bundesregierung findet keine Alternativen zu Uploadfiltern, Handelsblatt v. 17.9.2019, https://www.handelsblatt.com/politik/ deutschland/eu-urheberrecht-streit-um-artikel-17-bundesregierung-findet-keine-a lternativen-zu-uploadfiltern/25020802.html (alle Webseiten zuletzt abgerufen am 20.7.2020).

Banse, Philip, Das Internet - ein rechtsfreier Raum?, https://www.deutschlandfunk. de/das-internet-ein-rechtsfreier-raum.724.de.html?dram:article_id=99567.

Becker, Maximilian: Von der Freiheit, rechtswidrig handeln zu können, ZUM (2019), S. 636-648.

Beuth, Patrick, Umstrittene Urheberrechtsreform - Woher die Uploadfilter kommen könnten, Spiegel v. 6.4.2019, https://www.spiegel.de/netzwelt/web/upload-f ilter-fuer-die-urheberrechtsreform-wer-entwickelt-die-software-a-1261320.html.

Boldrin, Michele / Levine, David K., Against Intellectual Monopoly, first paperback edition, Cambridge, MA 2010.

Doctorow, Cory, Lockdown, https://boingboing.net/2012/01/10/lockdown.html.

74 Helfrich und Heinrich-Böll-Stiftung (Hg.) Commons Für eine neue Politik jenseits von Markt und Staat; abrufbar unter https:/www.boell.de/sites/default/files/ 2012-04-buch-2012-04-buch-commons.pdf (zuletzt abgerufen am 20.07.2020). 
Doctorow, Cory, Why do you give away your books?, https://craphound.com/littlebr other/about/\#freedownload/.

Dredge, Stuart, Why is the music industry battling YouTube and what happens next?, The Guardian v. 20.5.2016, https:/www.theguardian.com/technology/201 6/may/20/music-industry-battling-google-youtube-what-happens-next.

Freund, Wieland, „Das Internet ist ein rechtsfreier Raum“, Interview mit Alexander Skipis vom Börsenverein des Deutschen Buchhandels, Welt v. 15.4.2009, https:// www.welt.de/kultur/article3558839/Das-Internet-ist-ein-rechtsfreier-Raum.html.

GEMA, VG Bildkunst, VG Musikedition und VG Wort, Lizenzen vs. Uploadfilter, https://www.vgwort.de/fileadmin/pdf/allgemeine_pdf/Aktuelle_Information_Li zenzen_vs._Uploadfilter.pdf.

Goller, Marion. Copyfraud und Overclaiming - was beim Umsetzen der EU-Urheberrechtsreform zu beachten ist, https://irights.info/?p=29603.

Hans-Brewdow-Institut, Trumps Gesetz gegen Soziale Medien, Interview mit Dr. Stephan Dreyer und PD Dr. Matthias C. Kettemann, https://www.hans-bredow-inst itut.de/de/blog/trumps-gesetz-gegen-soziale-medien.

Hansen, Sven, Un-CD-Bändiger: Abspielprobleme beseitigen mit unCDcopy, heise online v. 5.4.2004, https://www.heise.de/ct/artikel/Un-CD-Baendiger-289314.ht $\mathrm{ml}$.

Hofmann, Franz: Prozeduralisierung der Haftungsvoraussetzungen im Medienrecht - Vorbild für die Intermediärshaftung im Allgemeinen?, ZUM (2017), S. 102109.

Hofmann, Franz: Fünfzehn Thesen zur Plattformhaftung nach Art. 17 DSM-RL, GRUR (2019), S. 1219-1229.

Hurtz, Simon, Upload-Filter löschen Dutzende Kopien des Mueller-Berichts, Süddeutsche Zeitung v. 21.4.2019, https:/www.sueddeutsche.de/digital/upload-filte r-mueller-bericht-scribd-1.4416802.

Kaiser, Ulrich, Von einem, der auszog, das Fürchten zu lernen..., https://blog.wikim edia.de/2018/08/06/von-einem-der-auszog-das-fuerchten-zu-lernen/.

Kastl, Graziana: Filter - Fluch oder Segen?, GRUR (2016), S. 671-678.

Koreng, Ansgar, Die Schutzfrist als Sichtschutz: Wie das Urheberrecht freies Wissen behindert, https://irights.info/?p=28237.

Kreutzer, Till, Das Modell des deutschen Urheberrechts und Regelungsalternativen, 1. Aufl., Baden-Baden 2008.

Kublen, Rainer, Erfolgreiches Scheitern - eine Götterdämmerung des Urheberrechts?, 1. Aufl., Boizenburg 2008.

Lee, Timothy B., How YouTube lets content companies “claim” NASA Mars videos, https://arstechnica.com/tech-policy/2012/08/how-youtube-lets-content-companie s-claim-nasa-mars-videos/.

Leistner, Matthias / Metzger, Axel, Wie sich das Problem illegaler Musiknutzung lösen lässt, Frankfurter Allgemeine Zeitung v. 4.1.2017, https://www.faz.net/aktue 11/feuilleton/medien/gema-youtube-wie-sich-urheberrechts-streit-schlichten-liesse -14601949.html. 
Leistner, Matthias / Metzger, Axel: The EU Copyright Package: A Way Out of the Dilemma in Two Stages, IIC (2017), 381-384.

Lessig, Lawrence, The Future of Ideas, 1. Aufl., New York 2002.

Lobo, Sascha, Streit über die Urheberrechtsreform - Wollt ihr Europa zerstören?, Spiegel v. 13.2.2019, https://www.spiegel.de/netzwelt/netzpolitik/urheberrechtsr eform-wollt-ihr-europa-zerstoeren-a-1252993.html.

Lohmann, Fred von, YouTube's Content ID (C)ensorship Problem Illustrated, https: //www.eff.org/de/deeplinks/2010/03/youtubes-content-id-c-ensorship-problem.

Metzger, Axel: Regulierung im Urheberrecht - Herausforderungen und Perspektiven, ZUM (2018), S. 233-242.

Nolte, Georg: Drei Thesen zur aktuellen Debatte über Haftung und Verteilungsgerechtigkeit bei Hosting-Diensten mit nutzergenerierten Inhalten, ZUM (2017), S. 304-312.

Ostrom, Elinor / Hess, Charlotte, Introduction: An Overview of the Knowledge Commons, in: Ostrom / Hess, Understanding Knowledge as a Commons, Cambridge (MA) 2007, S. 3-26, abrufbar unter http://www.wtf.tw/ref/hess_ostrom_2007. pdf.

Pachali, David, Kopiervergütung: VG Wort darf nicht pauschal an Verlage ausschütten, https://irights.info/?p=27286.

Schieferdecker, Daniel, Einfach mal Mäzen werden, Die Zeit v. 19.5.2020, https://ww w.zeit.de/kultur/musik/2020-05/patreon-musiker-crowdfunding-corona/komplet tansicht.

Specht, Louisa: Beschränkung der Verkehrsfähigkeit digitaler Güter durch technische Schutzmaßnahmen, ZGE / IPJ 8 (2016), S. 280-303, doi: 10.1628/18672371 6X14652041936314.

Specht, Louisa: Zum Verhältnis von (Urheber-)Recht und Technik - Erfordernis eines Dualismus von techniksensitivem Recht und rechtssensitiven technischen Durchsetzungsbefugnissen, GRUR (2019), S. 253-259.

Spindler, Gerald: Upload-Filter: Umsetzungsoptionen zu Art. 17 DSM-RL Vorschläge zur "Quadratur des Kreises“ im nationalen Recht, CR (2020), S. 50-59

Stieper, Malte: Ein angemessener Interessenausgleich im Verhältnis von Kreativen zu Rechteinhabern und Verwertungsgesellschaften?, ZUM (2019), S. 393-400.

Travis, Hannibal: Pirates of the Information Infrastructure: Blackstonian Copyright and the First Amendment, Berkeley Technology Law Journal (2005), Vol. 15, No. 777, 2000, abrufbar unter https://ssrn.com/abstract=758885.

Willman, Chris, Exclusive: Taylor Swift on Being Pop's Instantly Platinum Wonder... And Why She's Paddling Against the Streams, https://www.yahoo.com/ent ertainment/bp/exclusive--taylor-swift-on-being-pop-s-instantly-platinum-wonder---and-why-she-s-paddling-against-the-streams-085041907.html.

Wimmers, Jörg / Barudi, Malek: Der Mythos vom Value Gap - Kritik zur behaupteten Wertschöpfungslücke bei der Nutzung urheberrechtlich geschützter Inhalte auf Hosting-Diensten, GRUR (2017), 327-338. 